\title{
Skepticism about Reasoning
}

Sherrilyn Roush, Kelty Allen, and Ian-Cadoc Herbert

Less discussed than Hume's skepticism about what grounds there could be for projecting empirical hypotheses is his concern with a skeptical regress that he thought threatened to extinguish any belief when we reflect that our reasoning is not perfect. The root of the problem is the fact that a reflection about our reasoning is itself a piece of reasoning. If each reflection is negative and undermining, does that not give us a diminution of our original belief to nothing? It requires much attention to detail, we argue, to determine whether or not there is a skeptical problem in this neighborhood. For consider, if we subsequently doubt a doubt we had about our reasoning, should that not restore some confidence in our original belief? We would then have instead an alternating sequence of pieces of skeptical reasoning that cancel each others' effects on our justification in the original proposition, at least to some degree. We will argue that the outcome of the sequence of reflections Hume is imagining depends on information about a given case that is not known a priori. We conclude this from the fact that under three precise, explanatory, and viable contemporary reconstructions of what this kind of reasoning about reasoning could be like and how it has the potential to affect our original beliefs, a beliefextinguishing regress is not automatic or necessary. The outcome of the sequence of reflections depends on further information whose character we will explain.

Hume's discussion of this potential problem is highly telegraphic and reconstructing it requires a good bit of imaginative filling out. Our aim is to construct the strongest possible case for a skepticism in this area while remaining faithful to the letter of Hume's text, but these two goals will come into conflict, and we will not shy from suggesting that he had undefended assumptions and came to the wrong conclusion. After all, even Hume himself would not have claimed that his reasoning was perfect.

Hume describes a sequence of steps we are apparently reasonably led to take when reflecting on having been carried to a belief about an effect from observation of a cause. The first is a review of this act of the mind, a review that leads us to conclude that it is of an irregular nature and that the faculty that produced it is frail. Hume appears here to refer to the reasoning about inference between cause and effect that he used to generate the famous problem about induction, but we need not dwell on whether that is the precise reference; the reflections he describes seem equally applicable to demonstrative inference if we understand 
that as a fallible process, and ourselves as capable of seeing that. It is the further steps that present the new problem:

Having thus found in every probability, beside the original uncertainty inherent in the subject, a new uncertainty, derived from the weakness of that faculty which judges, and having adjusted these two together, we are obliged by our reason to add a new doubt, derived from the possibility of error in the estimation we make of the truth and fidelity of our faculties. This is a doubt which immediately occurs to us, and of which, if we would closely pursue our reason, we cannot avoid giving a decision. But this decision, though it should be favourable to our preceding judgment, being founded only on probability must weaken still further our first evidence, and must itself be weakened by a fourth doubt of the same kind, and so on in infinitum; till at last there remain nothing of the original probability, however great we may suppose it to have been, and however small the diminution by every new uncertainty. No finite object can subsist under a decrease repeated in infinitum; and even the vastest quantity which can enter into human imagination, must in this manner be reduced to nothing. Let our first belief be never so strong, it must infallibly perish, by passing through so many new examinations, of which each diminishes somewhat of its force and vigour. When I reflect on the natural fallibility of my judgment, I have less confidence in my opinions than when I only consider the objects concerning which I reason; and when I proceed still further, to turn the scrutiny against every successive estimation I make of my faculties, all the rules of logic require a continual diminution, and at last a total extinction of belief and evidence. [Hume, Treatise, Bk. 1 Pt. 4 Sec. 1]

We come to a strong but uncertain belief about something; the uncertainty in this belief is the First Doubt. Next by reasoning we judge the quality of our inference to that original belief. On thereby realizing the frailty of the faculty that judged - the Second Doubt - the force of our original belief reduces. However, we are further rationally required to reflect on the quality of that judgment we just made of the quality of the original inference. This reflection results in doubt about that estimation - the Third Doubt - due once again to awareness of the possibility of error. It is at the point of interpreting the consequences of this doubt that Hume's argument becomes hard to follow. The uncertainty added to the evaluation that led to diminution of the original belief may not be large - "the decision [c]ould be favorable to our preceding judgment" - but the fact that there is uncertainty at all leads, Hume says, to a weakening of our original belief. ${ }^{1}$

\footnotetext{
${ }^{1}$ We have changed "should" to "could" in this phrase because this was necessary to preserve the subjunctive meaning when quoting it out of context.
} 
It would seem that the new uncertainty, about the disparaging we had done of the faculty that led to our original belief, should restore somewhat our original belief, and this is an assumption that has been made without comment about Hume's argument. (Vickers 2000, 155156). Unfortunately, it flatly contradicts the text. The remainder of Hume's passage further confirms his meaning: "Let our first belief be never so strong, it must infallibly perish, by passing through so many new examinations, of which each diminishes somewhat of its force and vigor." (our emphasis) We will return to the question of how the uncertainty generated at a later stage in this sequence is intended by Hume to come back to be relevant to that original belief, and we will call this the Problem of Relevance. But it is clear so far that he thought every step of reflection diminished the force of the first belief, and also, we say, that it is reasonable to doubt that.

The doubt about that doubt of our faculties is of course also subject to doubt, Hume points out, and that is supposed further to diminish our first belief. This is the Fourth Doubt and the direction of the effect he thinks it has on our first belief makes some sense however the Third Doubt went, since any relief we might have gotten at that stage from the Second Doubt's disparagement of the faculty leading to our original belief would seem to be to some extent diminished by this doubt about the judgment that led to that relief, thus reviving at least some of the doubt about the original belief. At this point Hume takes it to be sufficiently clear how further steps are generated in a sequence that goes on ad infinitum, each stage involving an estimation of the previous estimation.

However, even if we grant Hume that every stage of doubt, including all odd-numbered ones, diminishes the original belief, there is another problem in his presentation having to do with what the eventual effect of that would be. In explaining why the infinite sequence of doubts leads to extinction of the original belief, Hume claims that "No finite object can subsist under a decrease repeated in infinitum." As stated this is not true, so one might wonder whether he is ignorant of the fact that there are decreasing series that converge to positive numbers. This was known to mathematicians by Hume's time, but Hume was not a mathematician. However, we think it is just as likely that he is assuming that the amount of the decrease in each stage is the same ${ }^{2}-$ with decreasing series that converge to positive values the amount of decrease diminishes at each stage, or diminishes by enough in alternation with increases, to prevent the series from converging to zero. But Hume gives no guidance as to why

\footnotetext{
${ }^{2}$ Of course, the extinction would also follow necessarily if each decrease was greater than the previous, or not sufficiently smaller, but these options are complicated enough that if Hume had them in mind we think he would have said so.
} 
we should think the amount of decrease is the same or otherwise at each stage. This highly consequential step of his argument depends on a comparative quantitative claim whose grounding is unclear. We call this and various issues like this that have to be ironed out to make sense of his argument the Problem of Quantity.

We saw a difficulty above in why the Third Doubt should affect the original belief in the direction of diminution rather than more intuitively provide a restoration, but whether the former or the latter is right it would need a justification. There is also a more general issue about why a realization of the possibility of error should automatically lead to a diminution of belief at any of these stages. The mere possibility of error does not imply what the direction of the error might be. That we might be wrong allows that the value we chose was too high or too low, or that our confidence was too high or too low. When we judge that our judgment that our reason is frail was subject to the possibility of error, we have not thereby determined that the error was an overestimation or underestimation of its frailty. If it is always an underestimation, then indeed provided the underestimation does not diminish with each stage it follows as Hume thought that every stage diminishes the force of our original belief. But if the direction of error alternated this would not follow. What tells us that the error is only possibly in one direction? If we cannot justify a direction at each stage, we cannot justifiably assert that each stage must give our original belief a diminution, or indeed that our original belief should change at all. We call this the Problem of Direction. Hume's argument depends on quantitative and directional assumptions that will need to be considered more carefully.

It should be clear that the quantitative and directional issues we will discuss here are not imposed on the subject matter by our, the authors', predilection for precise, formal, and often probabilistic descriptions, but rather are crucial to making sense of Hume's original argument. This should not surprise us because his talk of increase in uncertainty and decrease in force of belief, and his conclusion of extinction of belief in the infinite long run, are themselves quantitative. We are only lucky to live in a day when we have more developed tools for dealing with such issues.

We will begin with a discussion of problems we see in John M. Vickers' (2000) reconstructions of Hume's argument. This highlights the advantages of the three reconstructions we will offer, one by growing conjunction, another by confidence intervals, and a third by probabilistic re-calibration. We take it that the best reconstructions will solve the problems of Relevance, Quantity, and Direction while describing a coherent sequence of reflections that match the general contours of Hume's argument. The three reconstructions we 
describe achieve all of these things, and in particular show how extinction of belief in the limit could happen. However, in all three cases what actually happens in a given case depends on more information than can be justified a priori. Thus, we take issue with what Hume thinks the "rules of logic" require; we do not think there is a coherent sequence of skeptical reflections about one's previous reasoning that justifiably leads automatically to extinction of belief.

\section{Erosion and the Generic Weakness-of-Reason Predicate}

Vickers first reconstructs the effect of each member of the sequence of skeptical judgments Hume imagined via synchronic constraints on belief functions - claims about how beliefs should relate to each other at a given time. He finds an immediate contradiction in this approach, and concludes that the problem is the focus on a single time. This has plausibility since the skeptical reflections we are imagining take place sequentially in time, but we argue that his diachronic reframing of the matter is subject to virtually the same contradiction. We think also that his representation is not explanatory because it leaves too many crucial aspects to be written in by hand. We will argue that the real problem is the choice to express the estimation of one's reasoning in the previous step too generically, and as a proposition.

Vickers formulates the effect an observation that one's reason is weak should have on a belief one has whose reasoning source one is focusing on, via a constraint he calls Synchronic Erosion:

$b[X / b(X)=p \& W]<p \quad S E$

where $X$ is any proposition, $b()$ is the subject's belief function whose value, $p$, is a number between 0 and 1 inclusive, "/" means conditional on, " $\&$ " means and, and " $W$ " means the proposition that one's reason is weak. This says that the subject's belief in $X$ given that his degree of belief in $X$ is $p$ and he believes his reason is weak, should be less than $p$. (We assume, of course, that $b[b(X)=p \& W] \neq 0$.) The contradiction arises simply by substituting " $W$ " in for "X:"

$b[W / b(W)=p \& W]<p$

As long as $b[b(W)=p \& W]<p]>0$ the left-hand side of this inequality is 1 . But there is no degree of belief, $p$, that is greater than 1 . Thus, some revision of this picture is required. This 
should not be too surprising since it is unclear intuitively what erosion of one belief by another at a single time could be; erosion connotes a process.

To formulate the concept of erosion over time Vickers proposes Diachronic Erosion:

$b_{t+1}\left[X / b_{t}(X)=p \& W(t)\right] \neq p \quad$ DE

For example, the subject's degree of belief in $X$ at stage 1 , given that his degree of belief at stage 0 was $p$ and he now believes his reason was weak at stage 0 , should be different from $p$. Vickers now uses a weakness-of-reason predicate, $W(t)$, that is indexed to stage, and appropriately so since each skeptical judgment in the sequence is imagined as an estimation of the quality of the reasoning in the previous stage, which was different reasoning and possibly reasoning of a different kind than that in other stages.

Note that Vickers uses not equal to instead of the less than relation that would seem more appropriate to an erosion concept, and would dictate diminution in the force of belief in $X$ at every stage. Hume does not appear to see any confidence-increasing stages in his picture of the growing sequence of skeptical thoughts, but Vickers allows for that possibility, as indicated by his idea that the Third Doubt will increase one's degree of belief in $X$ above what the Second Doubt had done to it.

A contradiction in DE can be derived as above for SE, by substituting $W(0)$ in for $X$ $b_{1}\left[W(0) / b_{0}(W(0))=p \& W(0)\right] \neq p$

As long as $b_{1}\left[b_{0}(W(0))=p \& W(0)\right]$ does not equal 0 , we have that:

$b_{1}\left[W(0) / b_{0}(W(0))=p \& W(0)\right]=b_{1}[W(0) / W(0)]=1$

The value of $\mathrm{p}$ within the left-hand-side expression is irrelevant to the value of that expression. This makes DE inappropriate whether $p=1$ or not. If $p=1$ then DE implies that $p \neq p$. If $p$ does not equal 1 , then whatever the subject's degree of belief at stage 1 about what her degree of belief at stage 0 in the proposition $W(0)$ was, her stage 1 degree of belief in $W(0)$ must be 1 . The condition implies that she must be certain that her reason was weak, which is not consistent with the uncertainty that is supposed to attend every step - every belief, or at least every empirical belief which a belief about one's faculties is, should according to Hume have a First Doubt, an uncertainty. Contradiction and other problems in this representation cannot be avoided merely by moving to a diachronic treatment. 
There is a further reason to be dissatisfied with Vickers' representation, in our view. It is not explicit enough to be explanatory of why at any given stage our confidence in the original proposition should go up or down or by how much, and so offers a description rather than an explanation why the sequence leads to extinction of belief. This is easy to see when we draw out the cases of the Third and Fourth Doubts. (To avoid contradiction or trivialization we now assume that $X$ is not $W(0)$, in order to make the current point.) At the Third Doubt we reflect that our Second-Doubt reasoning in which we came to doubt our original uncertain belief was itself weak. At stage 0 our belief in $X$ was $p$,

$b_{0}(X)=p$

At stage 1 we revised this in light of a stage-1 belief that our stage-0 reason was weak.

$b_{1}\left[X / b_{0}(X)=p \& W(0)\right]=q \neq p$

That is, our new degree of belief at stage $1, q$, in the original proposition $X$ is a number different from that for $X$ at stage $0, p$, in light of the added belief that our reason was weak at stage 0 , that is, in light of the Second Doubt. This stage- 1 belief in $X$ then gets revised at stage 2 via the Third Doubt:

$b_{2}\left[X / b_{1}(X)=q \& W(1)\right]=r \neq q$

Our new degree of belief at stage 2 in $X, r$, is a number different from what the degree of belief in $X$ was at the last stage, $q$, because we now think our reason was weak in that last stage.

Notice that mathematically it is not forbidden that $r=p$. That is, as far as the formalization specifies, the Third Doubt might have restored our original belief to its original level, perfectly nullifying the Second Doubt. Should the restoration go that high or not? We might understand something of how a weakness claim would have a particular intensity of effect if the weakness claim itself had degrees of severity, but the $W(t)$ predicate does not have the structure to make that connection.

As far as these equations go, $r$ could actually go above $p$, a case in which the sequence of two doubts would make us more confident than when we began. This may not seem to make intuitive sense ${ }^{3}$, and Vickers rules it out independently, but he does so by stipulations that $q$ is

\footnotetext{
${ }^{3}$ This possibility makes intuitive sense in the following way: the subject realizes that in the Second Doubt about his reason, when he took the uncertainty in his initial belief to be too low, he was wrong. In fact, he missed that the error in the original belief was underconfidence. Instead of concluding that his original uncertainty was too low he should have concluded that it was too high, and raised the confidence in the original proposition.
} 
less than $p$ and $r$ is greater than $q$ and less than $p$. These are instances of the obvious general question how $p, r$, and $q$ should relate to each other, and why, and the equation does not reveal the answers. Hume's conclusion can be achieved by assuming that $q$ will be less than $p$ and $r$ will be less than $q$, since on his view every successive doubt is supposed to reduce our confidence in $X$, but Vickers thinks that $q$ is less than $p$ and $r$ is greater than $q$, since he thinks the Second Doubt should reduce faith in $X$ whereas the Third Doubt somewhat restores it. Either view is consistent with DE. Vickers achieves Hume's ultimate conclusion, that the original belief is eventually completely extinguished, by further stipulating that the extent to which the Third (and later odd-numbered) Doubt should restore confidence in $X$ is not greater than the extent to which the Fourth (and later even-numbered) Doubt should diminish it again, but this does not grow naturally out of anything in the representation either. It is not just that these further assumptions do not find any justification or explanation from the equations. Neither Hume nor Vickers give any argument at all for these comparative judgments.

It is of course possible that no equation can be written that is more explanatory, but obviously we think that is not so. We think that Vickers' explanatory difficulty - that the equation allows things to be Hume's way or Vickers' way depending - does illustrate a truth, namely that, contra Hume, the logic does not decide the direction or intensity of the effects of these doubts; we think more substantive information that will vary case by case is required for that. However, we do think there are logics for describing the situation that determine how the substantive information in a given case makes these effects go one way or the other, and our representations will identify and explain this.

Both of our complaints about Vickers' representation, that it leads to contradiction and that it is unexplanatory, can be addressed by indexing the reason-is-weak predicate to the proposition that it evaluated when it was (or was not) weak, or not using a predicate at all, as we have it below. We also represent the relation between a judgment that one's reasoning was weak and the confidence we should have in the conclusion that reasoning came to as a relation that has "moving parts." This explains the dependence between these judgments more deeply and addresses the Relevance Problem. The fact that when we use a predicate to express that reason is weak it is indexed to the proposition whose reasoning is under scrutiny makes it impossible to formulate the contradictory self-applications that Vickers ran into, and when we do not use a predicate at all even non-contradictory application of a predicate to itself is of course not possible. 
In the next three sections we present representations that we think are each able to capture the outlines of Hume's skeptical argument. When it becomes clear in each representation how the consequences of a doubt about a specific case of reasoning are determined, thus addressing the problems of Quantity and Direction, we see why and how particular substantive facts of the case determine whether a sequence of doubts should make us lose our belief entirely or somewhat, or oscillate between higher and lower confidence levels for eternity, or indeed converge to an increase in the force of our belief. All three of our pictures, which are otherwise quite different from each other, imply that the consequence for belief in $X$ of the sequence of skeptical doubts going on ad infinitum depends on substantive information that will vary with each case, suggesting the robustness of our conclusion that nothing about one's eventual confidence in the original proposition is dictated by the "rules of logic" alone in skeptical reflections about one's reasoning.

\section{The Account by Increase of Error over Lengthening Conjunctions}

Hume thought that confidence in one's original belief, $X$, should diminish with every step of the reasoning he described. The picture seems to be that uncertainty grows like an infection or a cloud gradually enveloping the whole proceeding, and hence its result, some belief-level or other in $X$. A plausible way to make sense of this is to think of the new judgment in each step of doubt as conjoined to the conjunction of the previous steps. It is well known that the probability of a conjunction strictly decreases as the conjunction increases in length by addition of conjuncts whose probabilities are less than one, that is, uncertain, as Hume does imagine all of them to be. This strict decrease is because the probability of a conjunction is a simple, multiplicative, function of the probabilities of its conjuncts. If the conjuncts are independent, then we multiply the probabilities of the conjuncts. If each conjunct might be wrong, then its probability is below 1 . Thus we are multiplying fractions of 1 and each new one lowers the probability of the conjunction. For this reason when additional fallible beliefs are conjoined to existing ones they strictly lower one's reliability about the whole set of beliefs. The fact that these probabilistic facts follow from the axioms would additionally explain Hume's impression that the diminution at every step was necessary rather than contingent, that it followed from the "rules of logic."

We will argue that this very plausible account will not yield Hume's conclusion. First, consider that the probability of $X$ is not decreased by the conjoining of independent propositions $Y$ and $Z$ to it. It is only that the probability of the new conjunction $X \& Y \& Z$ is lower 
than the probability of $X$. But the belief whose confidence is supposed by Hume to decrease as a result of each new skeptical reflection is the belief in $X$. Thus the structure of the doubting that Hume describes does not allow us to exploit the simple fact that longer, uncertain conjunctions have a lower probability. Mere addition of a conjunct does not change the probabilities of the existing initial conjunct, or of any other.

At least, this is so if the conjuncts are independent. However, though the new belief in $X$ that has to be less confident with each stage cannot be the conjunction of $X$ with the added doubts - it must be the belief in $X$ by itself - the whole point of these skeptical reflections is the sense that the legitimate level of confidence in $X$ is not independent of those doubts; somehow or other our legitimate belief in $X$ is supposed to depend on our judgment of the quality of our reasoning to it, and, by some as yet unexplained kind of transitivity relation, on our judgment of the quality of our reasoning to that, etc. What we just imagined was the accumulation of independent conjuncts, so we should not have expected it to work. Any successful representation must address the Problem of Relevance and the assumption that the new doubting judgments are independent of $X$ makes that impossible in one stroke.

Fortunately, the natural way of representing the dependence of confidence in $X$ on the quality of the reasoning to it, and the quality of reasoning to that, and so on, as a conjunction of interdependent propositions in a probabilistic framework is also the natural way, as we will see. However, in that account the resulting probability of $X$ is not calculated by the simple multiplication rule used for independent conjuncts. (Roush, manuscript a) As a consequence, decrease in confidence in $X$ is not a necessary result of each step, so the Humean conclusion is thwarted again.

We can see this by considering the relation of the evaluation of one's reasoning at a particular step to the confidence in what one had reasoned to at that step, as a conditional probability, and of the relation of the collection of judgments in the whole sequence as a disjunction of conjunctions. Assume that legitimate degrees of belief are probabilities, and let $R_{X}$ be the proposition that I reasoned well in coming to believe $X$, and $R_{X}^{\prime}$ be the proposition that I reasoned well in coming to believe $R_{x}$. The probabilities of these determine the probabilities of their negations. Now, we represent the dependence of $X$ on $R_{X}$, and of $R_{X}$ on $R_{X}^{\prime}$, and so on, as conditional probabilities, the probability of $X$ given that I did reason well to $X$, $P\left(X / R_{X}\right)$ and the probability of $X$ given that I did not $P\left(X /-R_{X}\right)$, and similarly for $R_{X}$ on $R_{X}^{\prime}$ and $-R_{X}^{\prime}$. I surely cannot know what my confidence in $X$ should be given my doubts about my reasoning if I do not know how that confidence depends on the verdicts of those doubts. In the cases of 
interest, my beliefs in $X, R_{X}$, and $R_{X}^{\prime}$ are uncertain - which means that all of the possibilities, $R_{X}$ and $-R_{X}$, and $R_{X}^{\prime}$ and $-R_{X}^{\prime}$, and the associated conditional probabilities, have positive (non-zero) and non-unitary probability values, and so must be taken into account.

Taking only the Second Doubt into account, the probability axioms give us the value of $P(X)$ as depending on the aforementioned probabilities and conditional probabilities, so:

$P(X)=P\left(X / R_{X}\right) P\left(R_{X}\right)+P\left(X /-R_{X}\right) P\left(-R_{X}\right)$

The formula of total probability, that follows from the axioms and of which this equation is an instance, represents these dependences in degrees and tells us that the probability of $X$ is an average of the probabilities of $X$ given that I reasoned well and that I did not, weighted by the probabilities that I reasoned well and that I did not. The summands each represent a possible state of affairs that itself consists of a conjunction of uncertain and interdependent states of affairs. We will say more about the intuitive meaning of the conditional probabilities presently.

My scrutiny of my reasoning to $X$ was my Second Doubt, and its result was recorded in a confidence (probability) that $R_{X}$, that is, that that reasoning to $X$ was sound, and in the likelihoods of $X$ given that $R_{X}$ and that $-R_{X}$. In the Third Doubt I scrutinize the reasoning of the Second Doubt, which will result in a confidence in $R_{X}^{\prime}$ - the claim that my reasoning (to a confidence for $R_{X}$ ) in the Second Doubt was sound. In the probabilistic representation, the formula representing how the resulting confidence in $X$ should depend on my confidence in the reasoning of my Second Doubt, is another instance of total probability, in which there is a disjunction of four conjunctions, representing the four possibilities: That I reasoned well to $X$, well to my evaluation of that, and well to my evaluation of that; that I reasoned badly to $X$ but well to my evaluation of that reasoning, and to my evaluation of that; that I reasoned well to $X$ but badly to my evaluation of that and badly to my evaluation of that too; and that I reasoned badly to $X$, badly about that reasoning, and badly in evaluating that too:

$$
\begin{aligned}
P(X)= & P\left(X / R_{X}\right) P\left(R_{X} / R_{X}^{\prime}\right) P\left(R_{X}^{\prime}\right)+P\left(X /-R_{X}\right) P\left(-R_{X} / R_{X}^{\prime}\right) P\left(R_{X}^{\prime}\right)+P\left(X / R_{X}\right) P\left(R_{X} /-R_{X}^{\prime}\right) P\left(-R_{X}^{\prime}\right)+P\left(X /-R_{X}\right) P\left(-R_{X} /-\right. \\
& \left.R_{X}^{\prime}\right) P\left(-R_{X}^{\prime}\right)
\end{aligned}
$$

The conditional probabilities $P\left(X / R_{X}\right)$ and $P\left(X /-R_{X}\right)$ represent what my confidence in $X$ should be if I did and if I did not reason well to $X$. My confidence that I reasoned well in coming to my confidence in $R_{X}, P\left(R_{X}^{\prime}\right)$, affects my confidence in $X$ only via the judgments of how my confidence in $R_{X}$ depends on my judgment of that reasoning, $R_{X}^{\prime}$ and how my confidence in $X$ should depend on my judgment of my reasoning to $X, R_{X}$. And this is faithful to intuition, for 
how could we know how my doubt about the evaluation of my initial reasoning should affect my confidence in $X$ unless we knew these dependencies? This is a straightforward and explicit representation of the judgments of interest to Hume, which captures and explains the dependence of my confidence in $X$ on my evaluation of my reasoning to $X$, and so on.

What Hume imagines is a process of doubting, of course, so we must replace these synchronic constraints with their diachronic counterparts, which are instances of Jeffrey Conditionalization:

$$
\begin{aligned}
P_{1}(X)= & P_{0}\left(X / R_{X}\right) P_{1}\left(R_{X}\right)+P_{0}\left(X /-R_{X}\right) P_{1}\left(-R_{X}\right) \\
P_{2}(X)= & P_{1}\left(X / R_{X}\right) P_{1}\left(R_{X} / R_{X}^{\prime}\right) P_{2}\left(R_{X}^{\prime}\right)+P_{1}\left(X /-R_{X}\right) P_{1}\left(-R_{X} / R_{X}^{\prime}\right) P_{2}\left(R_{X}^{\prime}\right)+P_{1}\left(X / R_{X}\right) P_{1}\left(R_{X} /-R_{X}^{\prime}\right) P_{2}\left(-R_{X}^{\prime}\right)+P_{1}(X /- \\
& \left.R_{X}\right) P_{1}\left(-R_{X} /-R_{X}^{\prime}\right) P_{2}\left(-R_{X}^{\prime}\right)
\end{aligned}
$$

These say how I get to a new confidence in $X$ after adding a new step of doubt. The function $P_{0}$ expresses my original degrees of belief before doubt of my reasoning began, at what we will call stage 0 . What Hume calls the First Doubt is expressed in the fact, as we will assume, that $P_{0}(X)$ is less than 1 . We assume that $P_{0}$ also has values for the likelihoods of $X$ given that $I$ reasoned well, and given that I did not. The first, $P_{0}\left(X / R_{X}\right)$, should be (roughly) the same as $P_{0}(X)$, since in accepting the results of the reasoning that gave me my degree of belief in $X, I$ expressed my pretty well full confidence in that reasoning. Hence, also, let us say for simplicity that $P_{0}\left(R_{X}\right)=1 .{ }^{4}$ The confidence I feel I should have in $X$ given that I made a reasoning error is not constrained by these other values. This probabilistic features mirrors the fact that I may feel I am more likely to have overestimated or underestimated if I erred, and logic that follows from generic doubt does not alone tell me which. In particular, if I think I am liable to have overestimated if I made a mistake, then $P_{0}\left(X / R_{X}\right)$ will be greater than $P_{0}\left(X /-R_{X}\right)$, and vice versa for underestimation.

The function $P_{1}$ is what the Second Doubt leaves me with; $P_{1}$ represents stage 1 . The Second Doubt gives me $P_{1}\left(R_{X}\right)$ and $P_{1}\left(-R_{X}\right)$, the absolute confidences that I did or did not reason properly in coming to my degree of belief in $X$. These values also involve information not supplied by logic alone. If $P_{1}\left(R_{X}\right)$ is 1 then the new function $P_{1}$ will be the same as $P_{0}$. This

\footnotetext{
${ }^{4}$ For presentational purposes we have left out the fact that this term cannot be 1 . The problem here is not that an extreme probability cannot change - as we will see in our set-up such changes happen exogenously anyway - but that the conditional probability $P_{0}\left(X /-R_{X}\right)$, needed for the conditionalization that gets us to $P_{1}(X)$, will be undefined on the usual axiomatizations of probability if $P_{0}\left(R_{X}\right)=0$. So, instead of 1 here and for $P\left(R_{X}\right)$ terms in all later stages we actually need $1-\varepsilon$, and instead of 0 we need $\varepsilon$. We have done the calculations to show that with this modification the results are as we advertize.
} 
mirrors the fact that if I am certain my reasoning was good, then I should have no inclination to change my confidence in $X$. On the assumption that the Second Doubt makes me withdraw some non-zero degree of certainty from $R_{X}$, I get from $P_{0}(X)$ to a new confidence $P_{1}(X)$ by using the aforementioned values via the first instance of Jeffrey Conditionalization given above. My new confidence in $X$ comes from a linear combination of my confidence about having made or not made a mistake and what the confidence-correction should be for $X$ were a reasoning mistake to have occurred.

For a case that behaves as we ordinarily expect, let our initial degree of belief in $X, P_{0}(X)$, be .9. At that same stage my confidence in $X$ given that I reasoned well to $X, P_{0}\left(X / R_{X}\right)$, is the same, .9 , and $P_{0}\left(R_{X}\right)=1$. Let us say that I also think that if I made a mistake it was a rather large overestimation; say $P_{0}\left(X /-R_{X}\right)$ is .6. At stage 1 I am taking into account that I came to my degree of belief in $X$ by reasoning and reasoning is fallible, so I am asking whether I did reason well to $X$. Suppose my confidence at stage 1 that I did, $P_{1}\left(R_{X}\right)$, is .8, implying that $P_{1}\left(-R_{X}\right)$, is .2. If all of this is so, then $P_{1}(X)=.84$. With these judgments about $X$ and myself my legitimate confidence in $X$ goes down from .9 to .84 in the transition from stage 0 to stage 1 . This happened because although I was more confident that I reasoned well than that I did not, I was not certain that I did, and I believed that if I had not then the direction of my error had been overestimation. Accordingly my confidence in $X$ came down, which matches well with intuitions under these assumptions.

The equation displays that the confidence in $X$ that results from the doubt about one's reasoning depends on the specific judgments that one made about quality of the previous reasoning; whether the doubt sends one's confidence in $X$ up or down depends on the strength and direction of the doubt, as can be seen in another example. Let $P_{0}(X)=.8$, and

$P_{0}\left(X / R_{X}\right)=.8$

$P_{1}\left(R_{x}\right)=.7$

$P_{0}\left(X /-R_{X}\right)=.95$

$P_{1}\left(-R_{X}\right)=.3$

In this case $P_{1}(X)=.85$, an increase in the subject's legitimate confidence in $X$, from .8 to .85 , despite the fact that she became newly uncertain that she reasoned well. Here she is still somewhat confident that she reasoned well, $P_{1}\left(R_{X}\right)=.7$, but she thinks that if she did make a mistake it was large $\left(P_{0}\left(X /-R_{X}\right)=.95\right.$, and $\left..95-.8=.15\right)$, and that it was in the direction of 
underestimation. She might have made a mistake, but if so it was in being underconfident about $X$, she thinks, so the results of the equation, a bumping up, are in line with intuitions.

Our question about the Third and subsequent odd-numbered Doubts, whether they necessarily issue in a decrease in confidence in $X$, is addressed similarly. The fact that the result may always go either way is expressed in the fact that no matter how many primes we put on a claim that the previous stage's reasoning was sound, $R_{X}$, the likelihood $P\left(X /-R^{n} X\right)$ is independent of other terms and may be either less than or greater than the likelihood $P\left(X / R^{n} x\right)$, issuing in a contribution of decrease or increase to the final confidence in $X$. This means that any sequence of changes in either direction and of any (non-extreme) degree is possible under this kind of doubting continued to infinity. This includes Hume's sequence of decrease at each stage, which would require simply that $P\left(X /-R^{n} x\right)$ is less than $P\left(X / R^{n} x\right)$ for every $n$, and that the amount by which it is less is not decreasing.

Errors in reasoning can in principle be in either an overestimating or underestimating direction and of any degree, so any equation that describes the logic of this sequence of reflections should account for both types of possibilities and determine how the outcome depends on the directions and relative quantities of the judged potential errors. This the equation of total probability and Jeffrey Conditionalization do, and they do so in the intuitively natural way. What happens in the long run depends on the nature of the evidence that prompts each new conditionalization, and thanks to first-order Bayesian convergence theorems we know that if the evidence stream is good, then conditionalization applied an infinite number of times will take one's confidence to the true probability.

There is a more precise way of seeing that each of the revisions requires substantive information. In the transition from $P_{0}$ to $P_{1}$ in the previous two examples we went from $P_{0}\left(R_{X}\right)=$ 1 to $P_{1}\left(R_{X}\right)=.8$ in the first, and from $P_{0}\left(R_{X}\right)=1$ to $P_{1}\left(R_{X}\right)=.9$ in the second. Both indicated a decrease in confidence that your reasoning was sound. This change is not expressed in the system - it is exogenous - but once effected the change in confidence in $R_{x}$ distributes itself across all relevant propositions. It is analogous to the way in which an observation changes a confidence in an observation statement, which is likewise followed by conditionalization on that change. Something prompts us to withdraw the implicit total confidence about our reasoning to $X$ that was expressed in stage 0 in our taking on the degree of belief in $X$ that that reasoning recommended. Perhaps it is the thought that all reasoning is fallible, or specific evidence about you as an individual, or what you did this time. Either way, $P_{1}\left(R_{X}\right)$, and the 
likelihoods $P_{0}\left(X / R_{X}\right)$ and $P_{0}\left(X /-R_{X}\right)$ must have values, or at least a range and ordinal relationship, or else no conditionalization is possible.

One might worry that our argument that a belief would not necessarily - through logic alone - diminish to extinction under skepticism about one's reasoning is circular. Must we not already be assuming a probabilistic interpretation of the skepticism when we claim that substantive information about degree and direction of potential error must be supplied in order to carry through the doubt? Moreover, is the probabilistic representation not completely unrealistic in describing the doubter as having that specific kind of information about his errors: their probability, degree, and direction? If the probabilistic representation requires the doubter to be so quantitatively informed in order to have any effect from his doubt, does that not count against the probabilistic interpretation? Doubting is surely easier than all that, and a sequence involving that much information may not even look like doubting.

Our conclusion that logic alone does not determine the eventual fate of the initial belief $X$ does not depend on probabilism - although a probabilistic representation illustrates it very well - but on the simple fact that an erroneous confidence, the kind of confidence that weak reasoning might have led one to, may be wrong in either of two different directions, that of over- and underconfidence. "Extinction of belief," we take it, means a withdrawal of confidence, or something analogous, so if one asserts as Hume does that a withdrawal of confidence in $X$ should happen in every step of the doubting, one is undeniably assuming that the suspected error is always one of overconfidence. Since underconfidence is a logical possibility, the grounds for this assumption must be extra-logical. And it is not that we never have such information, but it comes from our track record in reasoning and other such empirical considerations, not from the generic observation that we sometimes make mistakes.

There is a dilemma here for one who would defend the purely logical necessity of a skeptical regress to extinction. Hume thinks that belief in $\mathrm{X}$ should diminish with each stage of doubt. If anything could defend this idea of only decreases, it would be the thought that in general when we reflect that our reasoning is not perfect we should become a little less sure of its results. However, an application of this principle would force the Third Doubt to reduce confidence in what the Second Doubt delivered. The Second Doubt delivered a reduction in confidence in $X$, so that effect must be reduced. As a result, the Third Doubt would bring an increase in the confidence in $\mathrm{X}$ over what the Second Doubt had given it (as Vickers assumed above). The general principle that may seem to justify taking withdrawal of confidence in $X$ as the response to every doubt, properly applied actually exposes the fact that one needs 
substantive information to know the result of these doubts. For if odd-numbered doubts are raising, and even-numbered doubts lowering, the immediately preceding confidence in $\mathrm{X}$, then only if we have quantitative, or at least ordinal, information, will we be able to tell where the series ends up.

As for requiring the doubter to have too much information, we have already said that precise degrees of error attribution are not necessary - ranges or ordinal relationships are sufficient to proceed with Hume's type of reflections. But having information, or an assumption, about the direction of the suspected error is not optional, whether we use probabilities or not, as just explained. If one wonders how a person could have good reason to believe she is underconfident, one must also ask how a person could have good reason to believe she is overconfident. The latter is needed to take the skepticism in the direction Hume does. If we have insufficient information to identify at all what type of error we might be making then there simply is no further rational path for the skepticism to take. The probabilistic fact that without such information one cannot conditionalize to a new confidence in $\mathrm{X}$ is just a precise formulation of that point.

This representation is different from Vickers' in several ways. It is more explanatory because the dependences that dictate changes in one confidence because of changes in another are displayed explicitly, as conditional probabilities. For example, in Vickers' representation the equation governing change in confidence in $\mathrm{X}$ due to the Third Doubt is:

$b_{2}\left[X / b_{1}(X)=p \& W(1)\right] \neq p$

This states that the doubt about the reasoning at stage 1 should change the degree of belief in $X$, but does no more to explain why. Our equation by contrast says via a conditional probability statement, $P_{1}\left(X / R_{X}\right)=p$ for some $p$, that there is a probabilistic dependence between them. This dependence can also itself be broken down into moving parts, involving only conjunction and division, since $P(X / Y)=P(X \& Y) / P(Y)$. Thus we do not merely stipulate the relevance of one assessment to another but provide an explanation of it. That conditional probability is also a number, allowing an assessment of the degree and direction of the effect of $R_{X}$ on $X$. Such a conditional probability has a way of combining with $P_{1}\left(R_{X} / R_{X}^{\prime}\right)$, the effect of the next assessment on this one, to give a value for $X$ that takes into account both dependencies. The form of that combining is not simple transitivity, but it is completely governed by the more general axioms of probability alone and outputs a relation between the beginning and end of a sequence of whatever length we choose. 
We also note that in using conditional probability we are not representing the assessment of the quality of reasoning as a proposition. This allows us to avoid contradictions by application of a weakness-of-reason-predicate to itself, for there is no such predicate, only a conditional probability expressing how good or bad a judgment thinks the previous stage was via a conclusion of how confident we now should be in the result it gave us, if we take that judgment on board. Consider what it would be like to try to apply the total probability framework to the claim that a piece of reasoning was so and so weak as if that claim were $X$, the self-application maneuver we applied above:

$P_{1}(X)=P_{0}\left(X / R_{X}\right) P_{1}\left(R_{X}\right)+P_{0}\left(X /-R_{X}\right) P_{1}\left(-R_{X}\right)$

The subject's assessment of the reasoning to $X$ - how likely that reasoning makes $X$ - and hence the contribution of that judgment to the new legitimate confidence in $X$, is expressed by the judged value of $P_{0}\left(X / R_{X}\right)$ and since " $X / R_{X}$ " is not a proposition it cannot be substituted in for $X$. There is another aspect to the avoidance of self-application. To get a self-application, we might try substituting for $X$ the claim that the probability value is such and such, that is, substitute for $X$ the proposition $P_{0}\left(X / R_{X}\right)=p$, but that term does not show up in the condition of a conditional probability we use to update the confidence in $X$, as $W(t)$ did in:

$b_{t+1}\left[X / b_{t}(X)=p \& W(t)\right] \neq p$

Our updating equation does not take the form of one conditional probability. Thus, it seems to us that no matter how you think of it, self-application and the contradictions that it threatens do not arise in the representation we have just sketched.

\section{The Account by Confidence Intervals}

Beginning once again with the goal of explaining why Hume thinks confidence in $\mathrm{X}$ decreases with every step, and ends in extinction of belief, an account in terms of confidence intervals presents itself as a good candidate. In the use of the framework with data and parameter estimation in science, the original proposition is typically the claim that such and such a parameter, e.g. average height of corn plants, takes a certain value among a range of possible values. This number is not a level of confidence; whatever distance it is from 1 is not per se salient because this number is not a probability. It is, say, a corn height. Rather, in the confidence-interval picture our uncertainty is expressed by identifying a range around the specified parameter value that indicates where our information makes us think the value has a 
95\% chance of being. In cases where we are very confident, that interval is narrow: we are $95 \%$ sure the value is between, say, 45 and 46 inches. If we have a great deal of uncertainty, we are $95 \%$ sure, say, only that the value lies between 20 and 80 inches. It is easy to imagine reflecting on the $95 \%$ confidence interval we have identified and wondering about the quality of that assessment. The consequence of that might be a widening of the interval. We thought the value had a $95 \%$ chance of being between 45 and 46 , but each of those values is uncertain, so we must take the interval to be a little wider, must we not? Enough such reflections and we will be $95 \%$ sure only that the corn height is somewhere within the entire range of possible values.

A degree of belief is not a corn height, but the framework can be adapted. We are concerned with what our degrees of belief ought to be, so we will put the objective probability of $X$ in the place of the corn height. We start out thinking $X$ is probable; we are $95 \%$ confident that the probability lies between .8 and .85 . We do not have it down to a single value because there is uncertainty from the beginning - the First Doubt. But now, from an assessment of the fallibility of the tools and information we used to come to our original confidence we realize that any precise identification of the endpoints of that range would itself be subject to uncertainty, so those endpoints would have to be replaced with ranges, $95 \%$ confidence interval ranges. The endpoints of each of those precisely defined ranges would be subject to the same kind of uncertainty again, and the same after that. Since we are at each stage of reflection apparently replacing a point value with a range around that point value, it seems that this process can only make the confidence interval grow until at last the $95 \%$ confidence interval is 0 to 1 . This would be a kind of extinction of belief, though not a decrease in the level of confidence in the proposition. Rather, eventually we would be $95 \%$ confident only that the probability of $X$ is some value or other between zero and one. We do not feel we have any purchase on what its value is.

Despite the fact that the extinction process represented here does not match Hume's exact words about a decrease in confidence in $\mathrm{X}$, it does capture the arc of his argument in crucial ways. For example, the relevance of a reflection about one's reasoning about one's reasoning, etc. to one's original belief, comes naturally, as a widening of the confidence interval always pertains directly to a widening of the range of objective probability values one has confidence about for the original belief. Although judgments are made, and beliefs are formed, down the line of reflections about how wide that interval should be, there is no other belief that is represented in the picture. Only the upshots of those assessments are represented, as widenings of the intervals. For this reason it is also not possible to apply a predicate about the weakness of the preceding assessment to itself, for there is no such predicate. Thus we avoid 
contradictions of self-application. It also looks as if following to the infinite limit the process described here will necessarily lead to extinction as this representation depicts it. We need not know anything about a particular case, it seems, for the extinction follows from the rules of logic alone. [Figure 1 - moving left to right]
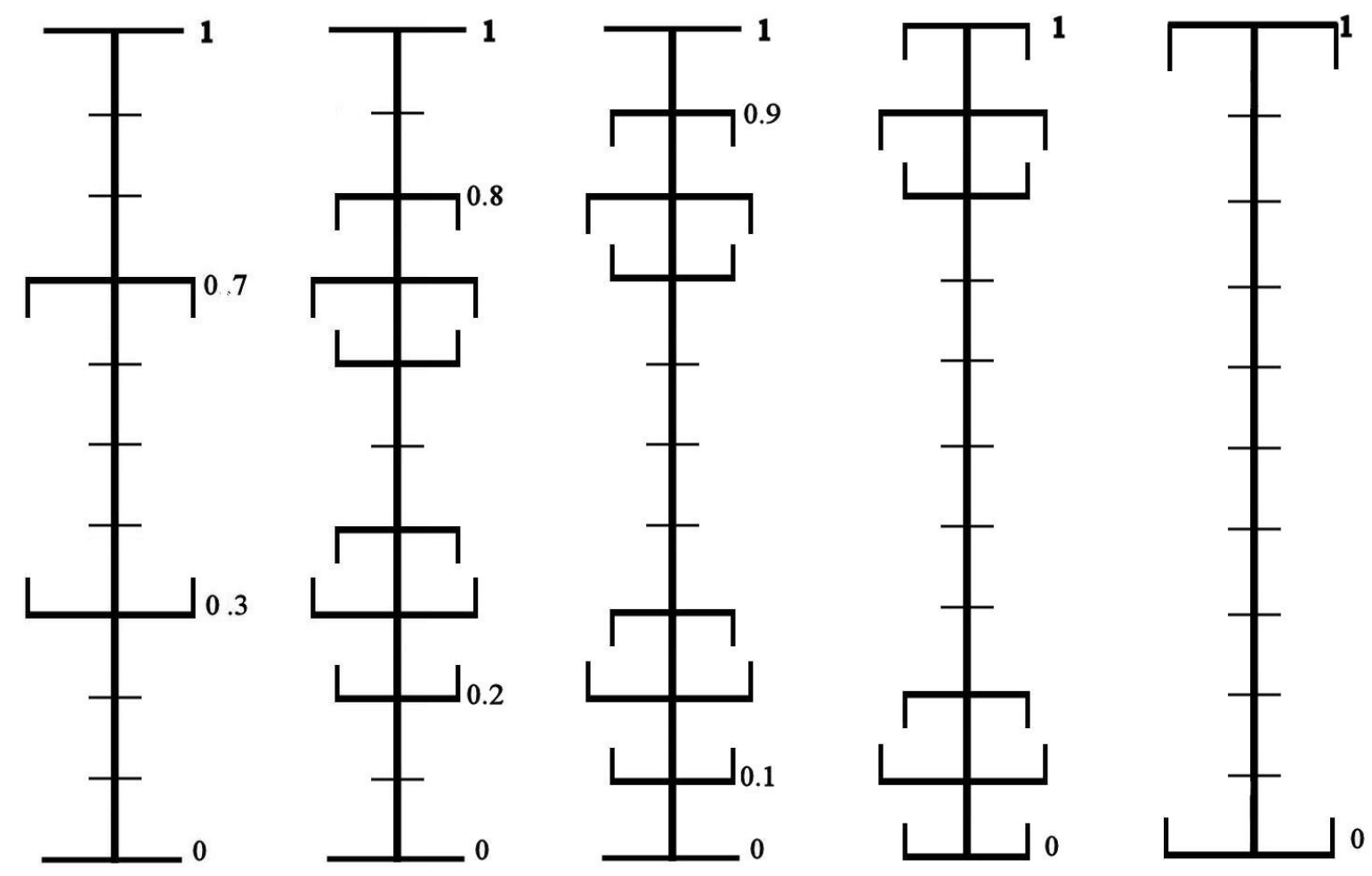

Unfortunately for Hume's version of the skepticism, and despite appearances, nothing follows automatically in this picture without assumptions about the quantity and direction of each newly discovered batch of potential error. The story and diagram just given are for a special case in which we presumed that the consequence of reassessing the interval we got to in the previous step is always a widening, both on the upper side and on the lower side, and by an amount which is not decreasing, or not decreasing fast enough, with each step. That this case exists does show that Hume's extinction-in-the-limit-idea is among the possibilities. It is not, however, necessary. Potential errors can, in general, come in either the direction of overshooting or the direction of undershooting, and as we have seen even both, in any given case. They can also come in any quantity, and these things go for errors in downgrading our confidence in our reasoning too. To get the case that we just saw, we had to presume that every error was in the direction of underestimating the uncertainty of the previous reasoning, and this by an amount that does not decrease (sufficiently) with each stage. If the amount of 
judged error decreased by half in each stage indefinitely, then the interval would converge to have endpoints somewhere less than 1 and greater than 0 respectively. [Figure 2]
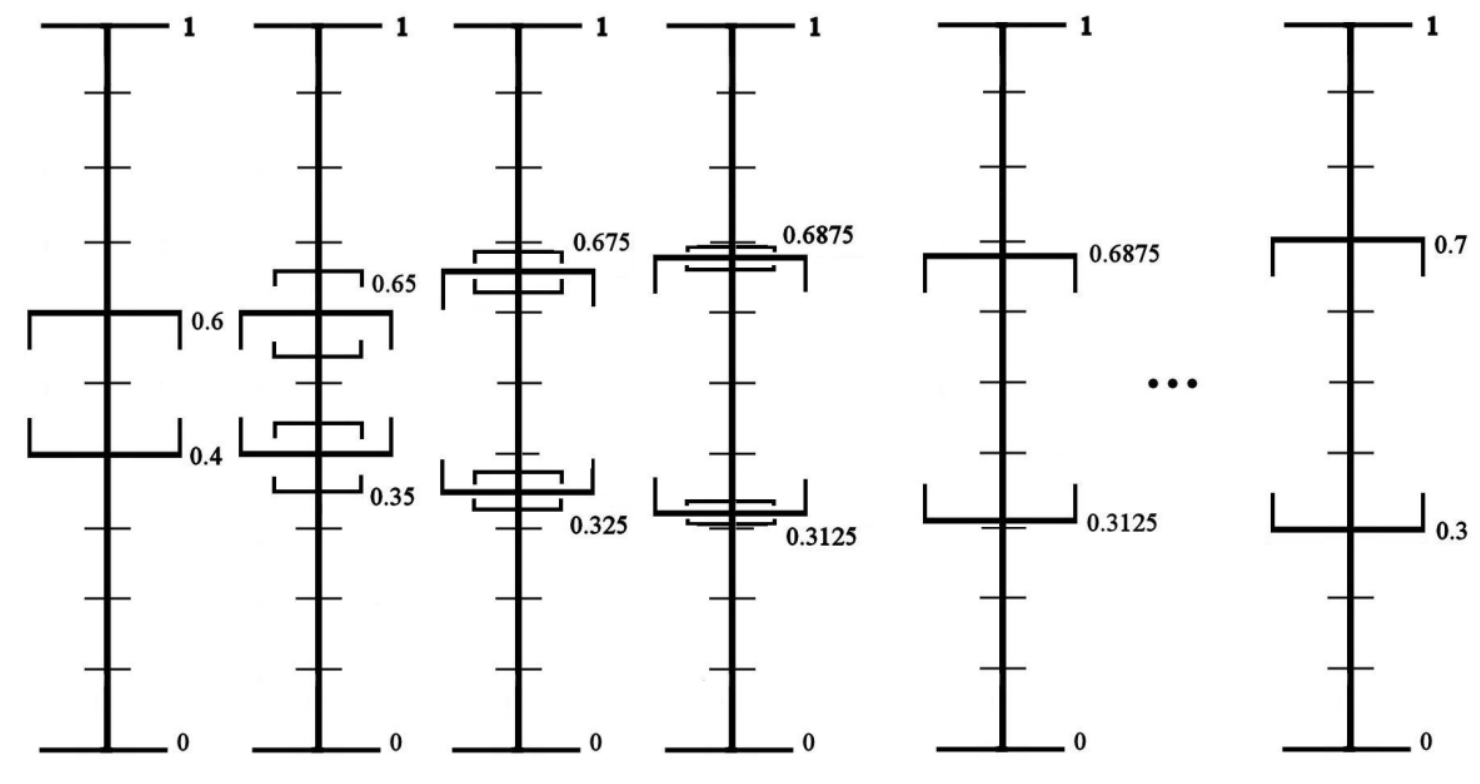

Obviously, an assessment of our previous reasoning could turn up that we took the 95\% confidence interval to be wider than we should have; that is also a way that the reasoning could have erred. If this was the direction of judgment at each step indefinitely, and the amount of error assessed was not decreasing, then the process would converge to a single precise value. [Figure 3] 

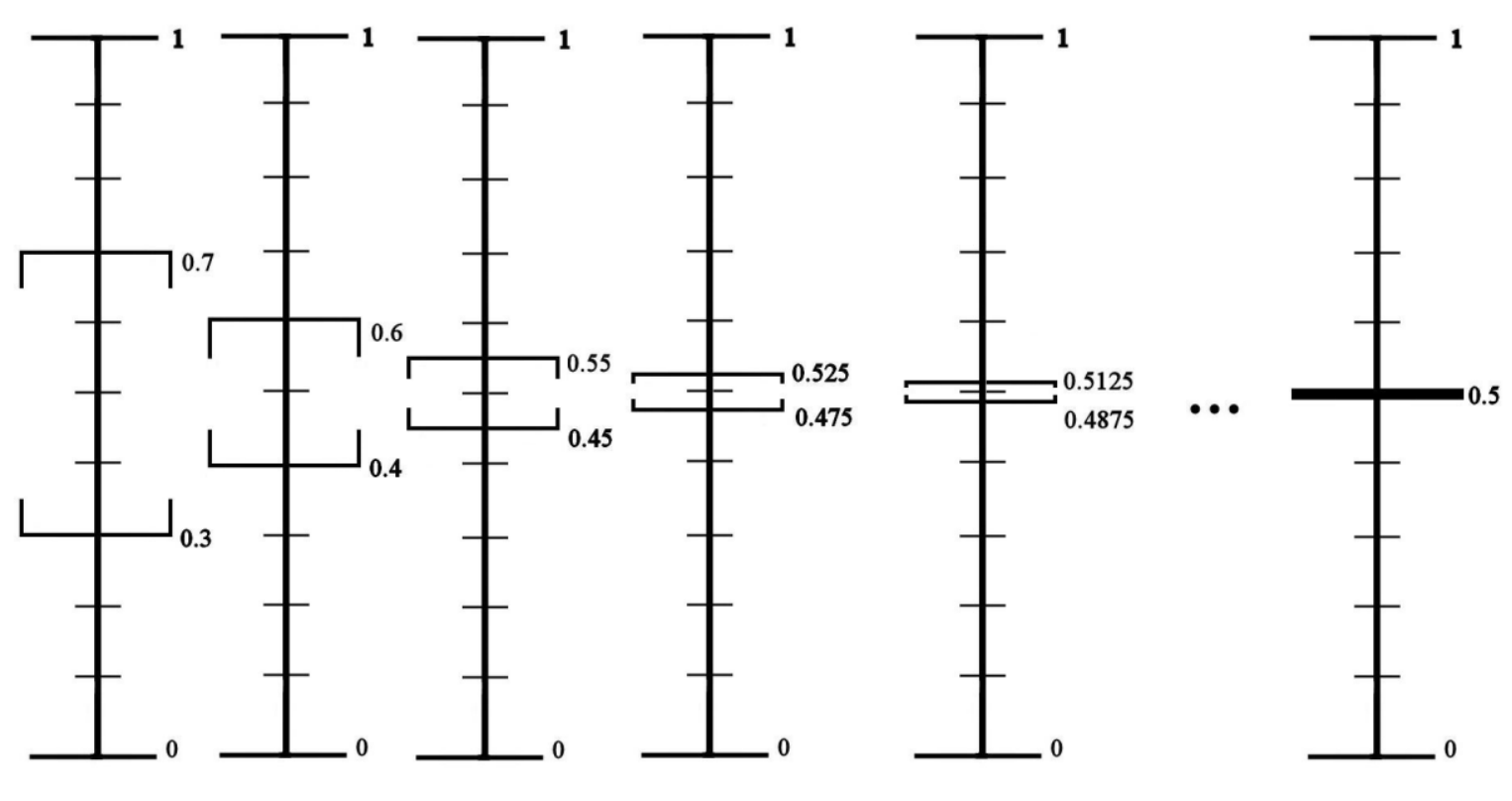

It is also possible that there is only reason to think that the interval needs to be widened in one direction. In this case, if the quantity of assessed error was not decreasing sufficiently at each stage then one would end up with an interval whose endpoints were either 1 or 0 at one end, and some non-extreme value at the other. [Figure 4] 

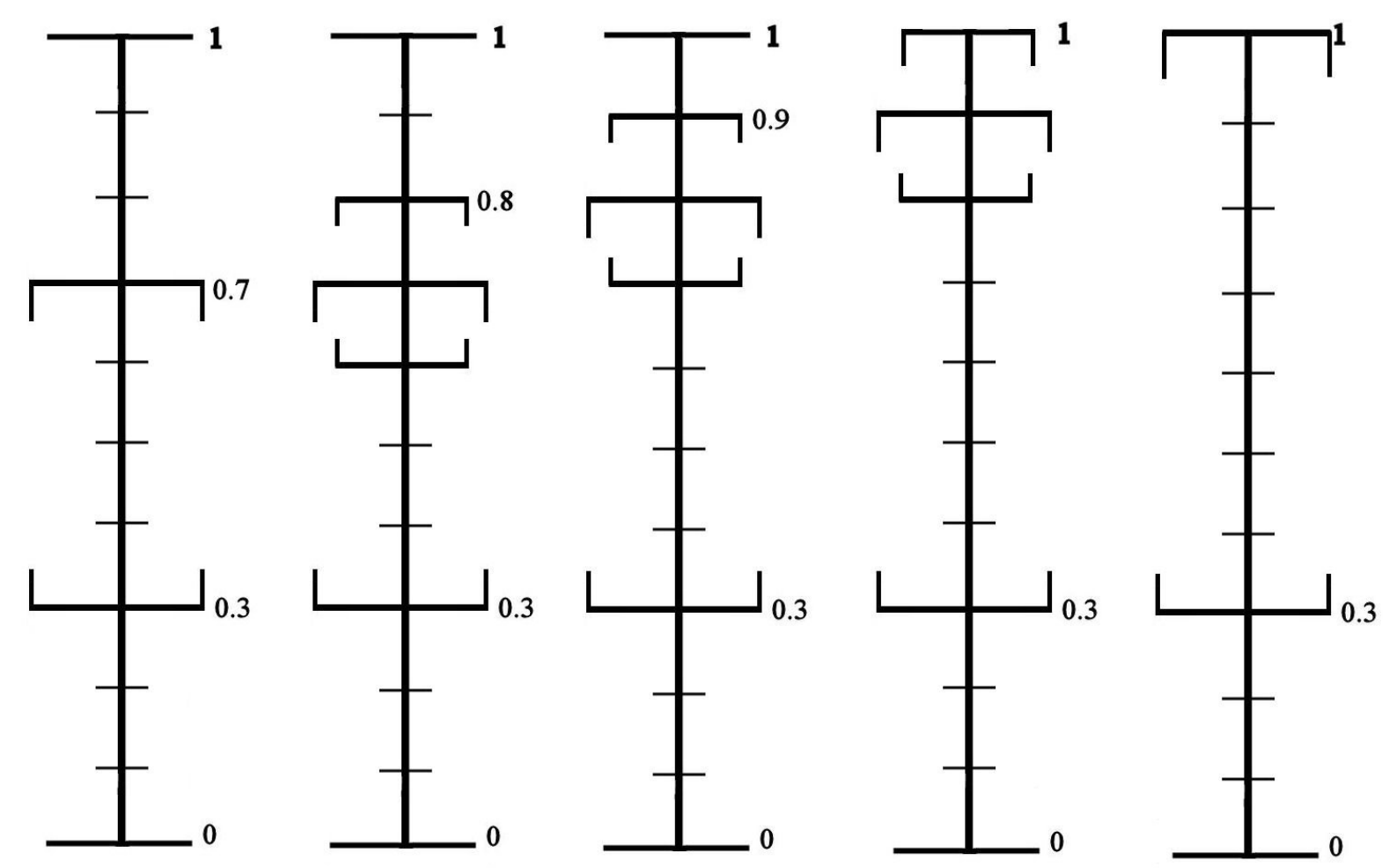

What these assessments of direction and quantity are cannot be dictated a priori, by logic alone, but depend on the substantive information available at each stage. Solving the interpretive Problems of Quantity and Direction exposes that the extinction here is not automatic. However, though the confidence-interval picture does not dictate what the eventual outcome of these reflections should be, it does give an explanation of how what those assessments are should affect belief in $X$, and in so doing explains the phenomenon we are concerned with.

\section{The Account by Probabilistic Re-calibration}

A sequence of reflections on our own beliefs and reasoning naturally lends itself to representation in terms of second-order subjective probabilities, i.e., degrees of belief about degrees of belief. We have seen that there are other compelling ways to represent it, but the existence of this one and the way it will turn out, lends further credence to our overall conclusion that the rules of logic do not necessitate extinction of belief in the long run of skeptical reflections about our reasoning. The representation we will use bears some 
resemblance to Vickers' Diachronic Erosion, but is more general, more deeply constrained and explanatory, and avoids the problems DE generated. ${ }^{5}$

In this representation we imagine the subject acquiring an explicit belief about the reliability of his process of coming to the degree of belief in $X$ that he finds himself with. This accords well with a passage from Hume's discussion:

We must, therefore, in every reasoning form a new judgment, as a check or controul on our first judgment or belief; and must enlarge our view to comprehend a history of all the instances, wherein our understanding has deceiv'd us, compared with those, wherein its testimony was just and true. [Hume, Treatise, 180]

He is clearly imagining measuring reliability of our reasoning by its track record in getting us to true beliefs. Here we represent the level of reliability of a way of coming to a particular degree of belief - in our case the evaluation of a piece of reasoning to that belief - as

$P R(X / P(X)=q)$

Where " $P R$ " means objective probability, of whatever sort one likes, and $P$ is a belief function that abides by the probability axioms. (Of course, of the objective interpretations of probability, the frequency interpretation of $P R$ is the one that would match Hume's words the best.) This definition says that the reasoning to the degree of belief $q$ in $X$ is more reliable the higher the objective probability of $X$ is when you come to degree of belief $q$ in $X$ by that means. It may seem odd that the term " $P R(X / P(X)=q)$ " can be high, say, .99, when your degree of belief $q$ is low, say .2 , and your degree of belief .2 will be called highly reliable in this case. .2 indicates that you have little confidence $X$ is true, whereas the .99 means $X$ is objectively probable, so how could your belief be reliable? The definition of reliability at this stage is not focused on a match between those two numbers, though - as we will see that is the quite distinct issue of calibration. The definition provides a measure of how far your degree of belief in $X, q-$ whatever $q$ may be - is an indicator of a high probability for $X$. Imagine your particular degree of belief as a flashing light of a particular hue. This definition is concerned with how likely $X$ is to be true when that hue of light is flashing, and has the virtue of not collapsing together the distinct issues of reliability and calibration.

\footnotetext{
${ }^{5}$ Vickers also presents an approach in terms of a notion he calls calibration, but we think it is unnecessarily complicated and yet not explicit enough to express what is needed.
} 
It also matches Hume's words as he asked how often when we found ourselves with a given, in his case high, degree of belief, reason has given us a belief in a truth. Low confidence in a truth is a symmetric error to high confidence in a falsehood, and the definition of reliability here captures all of those cases. Understandably, one may be much more concerned about one type of error than the other for practical reasons, but that does not define the logic.

The rule for updating in this framework when you come to believe that your degree of belief $q$ has a certain level of reliability is

$$
P_{f}(X)=P_{i}\left[X / P(X)=q \& P R\left(X / P_{i}(X)=q\right)=r\right]=r \quad \text { Re-Cal }
$$

When you find yourself believing $X$ to degree $q$ and think that your process in coming to that was reliable to degree $r$, then change your degree of belief to $r$. Hume's Second Doubt takes the form of the middle expression, with $f=1$ and $i=0$. We will justify Re-Cal's right hand side presently.

Reassuringly, although this rule can be applied ad infinitum to its own products mirroring Hume's regress of steps of doubt about your previous such steps, the iterative use of this updating rule does not involve probabilities or degrees of belief higher than the second order. This is because the rule's product is a new first-order degree of belief in $X$, in our case $P_{f}(X)=r$. To re-calibrate this re-calibrated degree of belief in $X$ is to do a merely second-order operation on it, which yields a new first-order degree of belief. There is never a need to have more than two " $P$ "s nested. ${ }^{6}$ This fact about the equation not only avoids a hierarchy of propositions practically impossible to contemplate, but also shows how each reflection is immediately relevant to one's confidence in the original proposition.

That next evaluation, the Third Doubt in our case, is done on a new belief-forming process, the one that got you to degree of belief $r$. That is, you will now evaluate how reliable you were, finding a value for the following term:

$P R\left(X / P_{1}(X)=r\right)$

What is the objective probability of $X$ given that you believe it to degree $r$ and came to that via the foregoing process (which involved both a coming to believe $X$ and a first re-calibration of that)? If you think that process might have given you the wrong level of confidence, then

\footnotetext{
${ }^{6}$ Worries about the coherence of applying a probability function to itself, of believing the condition in the conditional probability in Re-Cal, and of re-calibration being distorting or valueless are answered in Roush manuscript b. A few of these arguments are also made in Roush 2009.
} 
$P R\left(X / P_{1}(X)=r\right)=s$

for some s not equal to $r$. Now the updating rule tells you:

$P_{2}(X)=P_{1}\left[X / P_{1}(X)=r . P R\left(X / P_{1}(X)=r\right)=s\right]=s$

That is, change your confidence in $X$ to $s$.

This can go on ad infinitum, and where does it lead? The answer, as with the other frameworks, is that it very much depends on the substantive details of the judgments you make about the quality of the process that got you to a given belief. ${ }^{7}$ It depends most of all, at every stage, on the assessment of the reliability of the foregoing process, $P R\left(X / P_{i}(X)=q\right)$. Because at every stage the process and possibly the end product of it are different, every stage requires a new reliability judgment and potentially a change in one's confidence in $X$. One might come to think, at the Third Doubt, that in assessing one's original reasoning one got the direction of potential error right - say coming to too high a confidence - but overestimated how much that error was likely to have been. In this case, and in the language of Vickers' analysis above, $r$ is less than $q$, but $s$ is greater than $r$. The assumptions of this case would explain Vickers' intuition that the Third Doubt should restore some confidence in $X$. However, that is just a special case, and changing one of the assumptions about the case will yield Hume's intuition. So, imagine that in the Third Doubt one comes to the conclusion that one's reasoning about one's reasoning to $X$ did not disparage the former enough though it got the direction right. This would mean $s$ is less than $r$ in the reliability judgment, which translates directly to the degree of belief in $X$ going from $r$ to $s$, i.e. a decrease in confidence in $X$.

The equation Re-Cal does not merely identify new degrees of belief but also shows how the quantity and direction of one's assessment of one's previous reasoning at each stage determines whether one's degree of belief in X should go up or down, and that any quantity and either direction is possible; the matter is contingent. But how does it explain why an assessment of one's reasoning, and of one's reasoning about that, etc., should change one's degree of belief in X? How does it solve the Relevance Problem? The form of Re-Cal bears a resemblance to $D E$, in that the assessment of the reasoning of the previous stage $P R\left(X / P_{i}(X)=q\right)=r$ - stands there in the condition of a conditional probability, and appears to be

\footnotetext{
${ }^{7}$ As with first-order conditionalization, there is a proof that Re-Cal converges to the true probability of $\mathrm{X}$ when supplied with a stream of separating evidence. The method of proof is that due to James Hawthorne, in which convergence is defined as the likelihood ratio $\mathrm{P}(\mathrm{e} /-\mathrm{H}) / \mathrm{P}(\mathrm{e} / \mathrm{H})$ going to zero. This represents all alternative hypotheses to H having been eliminated by the evidence. See Hawthorne 1993, 1994, 2008, 2011, and Roush manuscript $b$.
} 
just stipulated to change one's degree of belief to $r$. However, there is a deep explanation of why one's new confidence should match one's judged reliability. Notice that the bolded condition in Re-Cal:

$P_{f}(X)=P_{i}\left[X / P_{i}(X)=q . P R\left(X / P_{i}(X)=q\right)=r\right]=r$

says that you believe $X$ to a certain degree and says that $X$ 's objective probability when you so believe is $r$. Under a natural assumption ${ }^{8}$ the first conjunct discharges the second conjunct to imply that the objective probability of $X$ is $r$. Consideration of the properties of your beliefs, expressed in the condition of that conditional probability, has given you a new assessment of the objective probability of $X$, namely that it is $r$. It is intuitive to think that your degree of belief in $X$ should match what you take the objective probability of $X$ to be, so your new degree of belief should be $r$. More technically,

$P_{f}(X)=P_{i}\left[X / P_{i}(X)=q \& P R\left(X / P_{i}(X)=q\right)=r\right]=r$

implies (under the natural assumption) the following:

$P_{f}(X)=P_{i}[X / P R(X)=r]=r$

and this is a diachronic instance of (a variant of) the Principal Principle, a constraint that says that our degrees of belief should match what we take the objective probabilities to be. ${ }^{9}$ Most people think some version of this principle is true and obvious. It has come under a great deal of discussion because there are obvious cases where it is false, and the task has therefore been to identify the principle's domain in a non-arbitrary way by admissibility conditions on any term that might be conjoined with " $P R(X)=r$ " in the condition. However, the cases in question in our topic are not of a type that produces obvious counterexamples, and no further conjunct of any sort is needed in the condition. Thus, it appears that the existing discussion of the domain of this principle can be carried over without any new challenges.

This relation of Re-Cal to the Principal Principle means that the relevance of an evaluation of our reasoning to our degree of belief in $X$ that is expressed in Re-Cal is not a mere stipulation. It is justified by a general principle of rationality that is independently compelling: we should aspire to have our degrees of belief conform to our beliefs about the objective

\footnotetext{
${ }^{8}$ The assumption is $P(P R(P(q)=x)=1 / P(q)=x)=1$, which is an instance of $P(P R(A)=1 / A)=1$. I.e., you are certain given $A$ that the objective probability of $A$ is 1 . It would be true if $P R$ is objective chance, but it is not trivial. ${ }^{9}$ The Principal Principle is typically formulated with objective chances in the place of our more general objective probability, $P R$. We think the principle is plausible for any objective notion of probability, and we intend Re-Cal to be useable with any of them.
} 
probabilities. Re-Cal is a way of getting oneself back into conformity with that principle when one has gotten out. This both solves the Relevance Problem and gives depth to the recalibration explanation of the revisions that our reflections about our reasoning should prompt in us.

Though Re-Cal somewhat resembles DE, it does not have the contradictions by selfapplication of weakness-of-reason predications, because application of the evaluation of a piece of reasoning to itself as a proposition cannot be formulated. This is not insured by stipulation either, but falls naturally out of the specificity we have demanded of the selfevaluation step. So, the proposition expressing the evaluation of a piece of reasoning to $X$ is $P R\left(X / P_{i}(X)=q\right)=r$. We can substitute this in for $X$ in the consequent of the equation and in the part of the antecedent (condition) that identifies our degree of belief in it:

$P_{f}(X)=P_{i}\left[X / P(X)=q . P R\left(X / P_{i}(X)=q\right)=r\right]=q$

To exploit the fact that $P(Q / Q)=1$, for any $Q$, to produce a contradiction, we must force ReCal's right hand side to be 1 by having the same expression in the condition and the consequent of the conditional probability. In DE we put $W(t)$ in for $X$ and had that in the condition too, to achieve this. However, in Re-Cal that $X$ also shows up within the reliability term, so when we substitute the reliability judgment in for $X$ in the consequent of Re-Cal as we just did, we cannot leave the second conjunct in the condition unchanged; that $X$ must be switched out too for a $P R\left(X / P_{i}(X)=q\right)=r$. This makes that second conjunct:

$$
\operatorname{PR}\left(\operatorname{PR}\left(X / P_{i}(X)=q\right)=r / P_{i}\left(P R\left(X / P_{i}(X)=q\right)=r\right)=q\right)=r
$$

which is not the same as the " $P R\left(X / P_{i}(X)=q\right)=r$ " we put in the consequent. ${ }^{10}$ Re-Cal thwarts us as we chase down self-applications because of the specificity the formula demands when it indexes the result to the proposition the reasoning to which is under scrutiny.

\footnotetext{
${ }^{10} \mathrm{Re}$-Cal does not make it easy to find instances of contradictory self-applications, but prima facie it is fair to expect that they exist in the form of propositions that effectively refer to themselves. For example, it seems that a fixed point might be constructible in which $\mathrm{X}$ was equivalent to the proposition $P R\left(X / P_{i}(X)=q\right)=r$. If so, then for that $X$ the left-hand side of Re-Cal would be 1 regardless of what q was, and Re-Cal would lead to contradictions. However, this is a problem that self-referring propositions present for any function on propositions, so it is not a problem specially imposed by Re-Cal. With Vickers' formula DE a contradiction could be generated simply by taking as the original judgment the statement that one's reason was weak, that is, by a simple substitution. No appeal needed to be made to the existence of sentences that are inherently paradoxical independently of the current discussion to generate that problem.
} 


\section{Necessary Truths and Falsehoods}

In two of our three representations of Hume's doubts - the account by lengthening conjunctions and the account by re-calibration - we apply probability to the proposition $X$. This raises the important question whether these frameworks can be used to understand doubts about beliefs we acquire concerning necessary truths, and the demonstrative reasoning that we can use to get to these and also to get from one empirical proposition to another. The probabilities of logically and mathematically necessary truths and falsehoods must be 1 or 0 respectively on pain of incoherence, and regardless of the subject's epistemic relation to these propositions, and because the probabilities are extreme they cannot be revised by standard forms of conditionalization.

Daniel Garber (1983) provided a way of representing learning of logical implications that could be helpful here. In this method, the language that gives the probability function its domain is reconfigured so that the logical truths of interest do not present themselves as such. For this reason they need not be assigned extreme probabilities, and their probabilities can change in response to epistemic changes in the subject. It appears that this could be adapted for our present case of revising confidences about logical truths and logical implications.

Hume's skeptical problem discussed here requires that we contemplate having reason to revise confidences in logical propositions. The prospects for coherently achieving this without hiding a proposition's status as a logical truth appear dim. Since probabilistic coherence requires a particular, unique degree of belief in each of these propositions -1 for truths, 0 for falsehoods - either the original confidence or the confidence resulting from the revision would render the subject incoherent (assuming the confidences are different). Revision of degrees of belief in logical propositions treated as such will lead to incoherence. However, incoherence, defined as violation of the axioms, may not be the end of the world. lan Hacking (1967) in describing an axiomatization of probability that allows non-extreme probabilities for these propositions made the point that although the subject becomes incoherent if he does not have full confidence in logical truths and zero confidence in logical falsehoods, he is not Dutchbookable. This is because one cannot assume the bookie knows more than the subject does rationality surely cannot require you to avoid losing to those who know more than you do. Here that general point means that the bookie cannot exploit the subject's lack of logical knowledge. 
If this kind of set-up were tolerated, then Re-Cal would make it very easy to model revising one's confidence in a logical proposition on the basis of reflections about one's reasoning. The rule is distinct from first-order conditionalizations in that even if one did have an extreme degree of belief in a logical proposition, the rule would allow one to change that. This is because the rule has one's confidence changing in response to evidence about what one's beliefs are, and what that indicates rather than conditioning on the proposition itself. For this reason the probabilistic irrelevance to every proposition of a proposition with an extreme probability does not trivialize the conditionalization. Intuitively, the process here would look just like it does for contingent propositions: one could come to believe that one is highly confident of a mathematical proposition (and might actually be certain of the mathematical proposition), find evidence that a person is generally wrong when coming to that the way one did (say, one acquires evidence that one's mathematical authority figure was actually Bozo the Clown in disguise), and because of those two things change one's confidence to zero or some other value.

If we are not logically omniscient, all that the axioms can do about it is tell us we are naughty. However, Re-Cal can recommend steps to take to get to coherence. This is analogous to the way it tells us how to get back in line with the Principal Principle when we have by our own lights fallen out. Obviously, this approach to logical propositions would require much further development than can be attempted here. In particular, although a convergence theorem is proven for the use of Re-Cal on contingent propositions (Roush manuscript $b$, Hawthorne 2011), it is unclear as yet how to prove such a thing for its use on necessary propositions.

\section{Conclusion}

The regress of skepticism that Hume offered concerning our reasoning is intuitively disconcerting. Doubt piles upon doubt because the worry at each stage is that our reasoning in the previous stage was weak, but - we soon discover -- this worrying stage is itself also reasoning. However, according to three otherwise quite faithful formal representations of Hume's argument, the perceived problem does not stand up to scrutiny because the outcome of such a sequence of doubts depends on substantive assumptions at every stage about the direction and degree of potential error, not, as Hume thought, on logic alone. It is possible for 
the infinite sequence of such reflections to lead to indifference about the original proposition that is, to "extinction of belief" - but nothing a priori excludes other possibilities, including that reflections on and evidence about the quality of our cognitive endeavors lead rationally to an increase in confidence.

\section{References}

Garber, Daniel (1983), “Old Evidence and Logical Omniscience in Bayesian Confirmation Theory," Testing Scientific Theories, Minnesota Studies in the Philosophy of Science, Volume X, John Earman ed,, 99-131.

Hacking, Ian (1967), "Slightly More Realistic Personal Probability," Philosophy of Science, Vol. 34, No. 4 (Dec., 1967), pp. 311-325

Hawthorne, James (1993) "Bayesian Induction is Eliminative Induction," Philosophical Topics, v. 21 , no. 1, 99-138.

(1994), “On the Nature of Bayesian Convergence," PSA 1994, v. 1, 241-249.

--------- “Inductive Logic," The Stanford Encyclopedia of Philosophy (Sept. 2004, revised Feb. 2008, major revision April 2011), Edward N. Zalta (ed.); pdf version available from the SEP, 131 pages. Online version at $\leq$ http://plato.stanford.edu/entries/logic-inductive/ $>$.

--------- (2011), Bayesian Confirmation Theory," Continuum Companion to Philosophy of Science, Steven French and Juha Saatsi, eds. London, New York: Continuum Press, 197213.

Hume, David (1888), A Treatise of Human Nature, L. A. Selby-Bigge, ed., Oxford.

Roush (2009), "Second-Guessing: A Self-Help Manual," Episteme, Vol. 6, No. 3, 251-268.

--------- (manuscript a), “Justification and the Growth of Error," Philosophical Studies, June 2012 online, forthcoming in print.

--------- (manuscript b), “Rational Self-Doubt: The Re-Calibrating Bayesian.”

Vickers, John M. (2000), "I believe it, but soon I'Il not believe it anymore: Scepticism, Empiricism, and Reflection," Synthese 124: 155-174. 